\title{
Shape Recognition of Nanoparticles by High-Resolution SEM and TSEM
}

\author{
E. Ortel ${ }^{1}$, L. Pellutiè ${ }^{2}$, F. Pellegrino ${ }^{2}$, V. Maurino ${ }^{2}$, J. Mielke ${ }^{1}$, B. Powierza ${ }^{1}$, I. Häusler ${ }^{1}$, W. Österle ${ }^{1}$ and \\ V.-D. Hodoroaba ${ }^{1}$
}

${ }^{1}$ BAM Federal Institute for Materials Research and Testing, Berlin, Germany.

${ }^{2}$ Department of Chemistry, University of Turin, Turin, Italy.

Engineered nanoparticles (NPs) are used as an active material in sensors, photovoltaics, photocatalysis, etc. Numerous publications have shown that particular facets of NPs dramatically influence their performance, e.g. in photocatalytic reactions with $\mathrm{TiO}_{2} \mathrm{NPs}[1,2]$. Therefore, information about the NP morphology expressed as area ratios of particular facets is highly demanded for the development of advanced nanomaterials.

The accurate determination of the particle size distribution for spherical NPs is a task resolved rather easily by various techniques. However, measuring the morphology of individual NPs having complex 3D geometries like cubes, prisms or (bi)pyramids is challenging. Often, only time-consuming TEM and TEM-tomography experiments can resolve the 3D structure and facets of particles in the nanometer range accurately. We present new approaches based on i) top-view high resolution SEM and ii) in-depth view, transmission SEM (TSEM) for the determination of the full shape of facet-controlled NPs.

From top-view high resolution SEM (approach i) we could identify the 3D geometry and the individual facet boundaries of NPs. A computer-generated 3D skeleton was manually matched to these facet boundaries, thereby yielding the necessary parameters for a full 3D description of the NP shape. The evaluation of the NP shape from TSEM (approach ii) relies on an automatic image analysis: The 2D projection of the particles on the image plane depends strongly on their orientation with respect to the incident electron beam. Taking only those particles into account for which the $2 \mathrm{D}$ projection coincides with an assumed silhouette for standing or lying NPs on the substrate, the size of the corresponding NP facet can be determined. Due to automated image analysis, statistically relevant amounts of data could be generated quickly and lead to reliable estimates of the NPs facet size.

These general procedures to determine the NP shape is demonstrated on truncated bipyramidal $\mathrm{TiO}_{2}$ anatase NPs. The $\mathrm{TiO}_{2}$ NPs were synthesized by a ligand-assisted synthesis route, which provides a scalable model system with tuneable $\mathrm{TiO}_{2} \mathrm{NP}$ geometries $[1,2]$. Figure 1 presents top-view SEM micrographs of $\mathrm{TiO}_{2}$ anatase NPs. The truncated bipyramidal shape is clearly recognizable in Figure 1A. An enlarged part of A shows a single NP where individual facet boundaries can be identified (Figure 1B). A computer-generated 3D skeleton was matched to the facet boundaries (Figure 1C) which results in a full 3D description of the NP shape (Figure 1D). Figure 2 consists of TSEM micrographs of the truncated bipyramidal $\mathrm{TiO}_{2} \mathrm{NPs}$ whereas $\mathrm{A}$ ) shows the original micrograph and $\mathrm{B}$ ) the same micrograph with NPs considered for the evaluation of the NPs shape. The NPs marked in red and yellow were identified as standing upright and flat-lying NPs on the support, respectively (Figure 2B). Hence the corresponding NPs lengths and widths could be measured from the TSEM micrograph.

In order to validate the new methods and estimate the associated measurement uncertainties, TSEM images of randomly oriented bipyramidal particles with known dimensions were simulated and evaluated in the same way as the measured images. A good agreement was obtained between the known parameters and the evaluation results. Furthermore, for selected NPs, the obtained dimensions were confirmed by HRTEM measurements.[3] 


\section{References:}

[1] C. Deiana et al., Phys. Chem. Chem. Phys. 15 (2013), p. 307.

[2] C. Li et al., J. Am. Chem. Soc. (2015), DOI: 10.1021/ja5111078.

[3] The research leading to these results has received funding from the European Union's Seventh Framework Programme (FP7/2007-2013) under grant agreements $\mathrm{n}^{\circ} 604577$ (SETNanoMetro) and $\mathrm{n}^{\circ} 604347$ (NanoDefine).
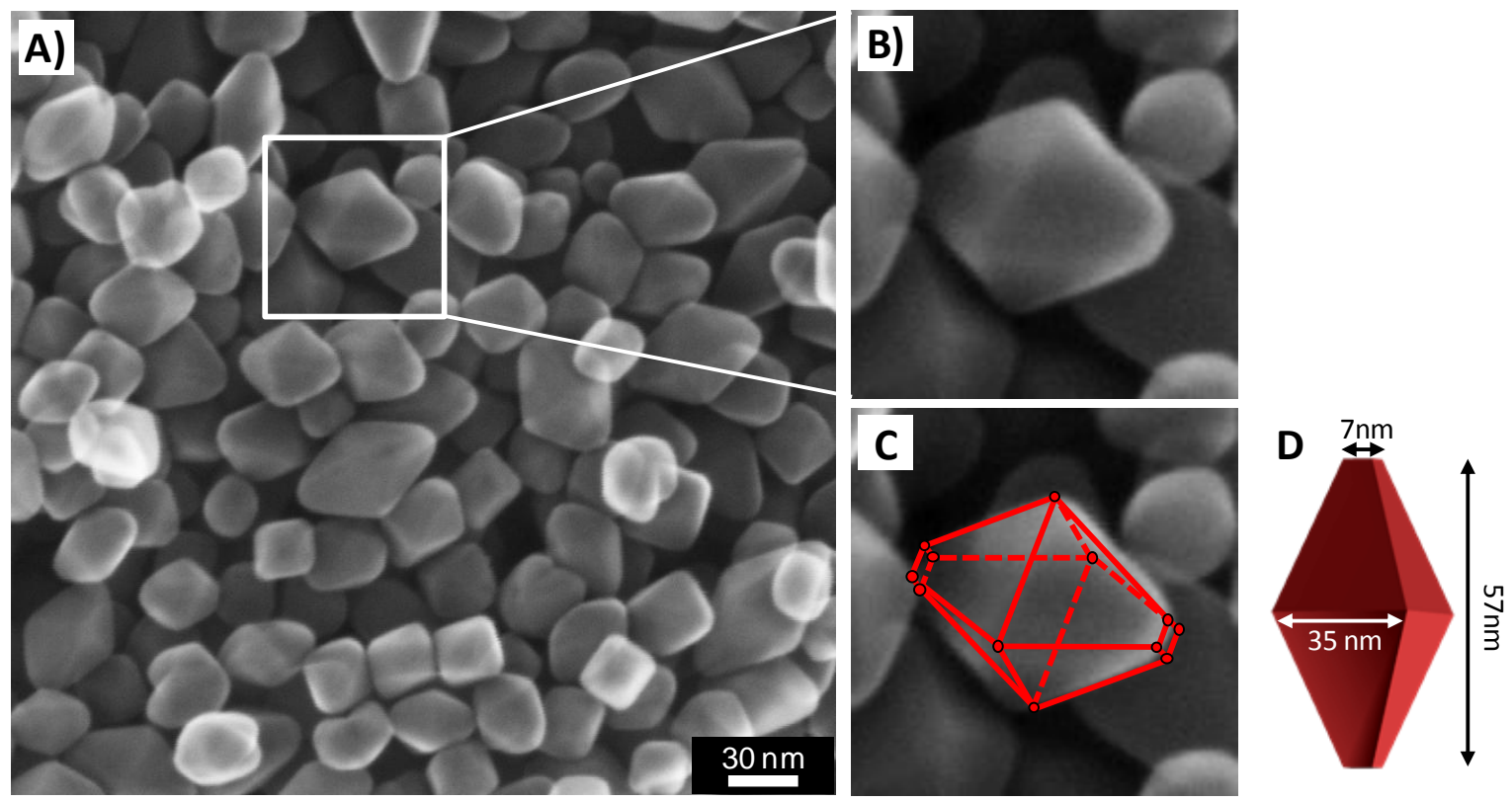

Figure 1. Top-view SEM micrographs of truncated bipyramidal $\mathrm{TiO}_{2} \mathrm{NPs}, \mathrm{A}$ ) overview, B) zoom-in on a single NP where individual facet boundaries can be identified, C) computer-generated 3D skeleton matched to facet boundaries D) full 3D description of the NP.
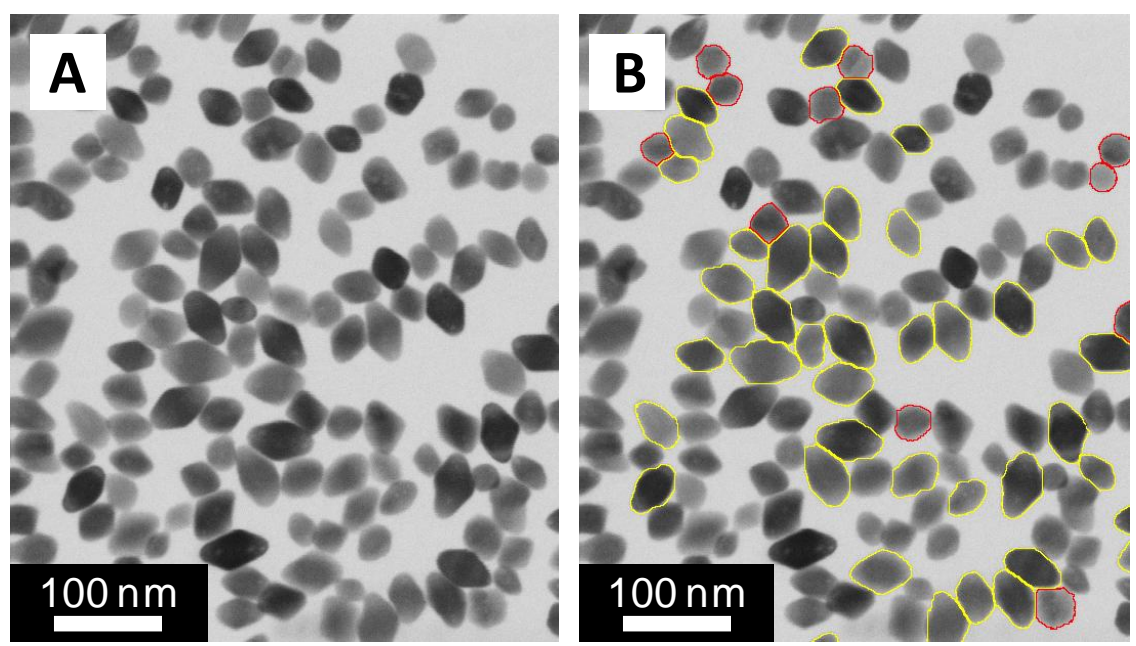

Figure 2. TSEM micrograph of truncated bipyramidal $\mathrm{TiO}_{2} \mathrm{NPs}$, A) original micrograph, B) NPs considered for the evaluation of NPs widths (red boundaries) and lengths (yellow boundaries). 